\title{
Research Article \\ Exact Solutions and Localized Structures for Higher-Dimensional Burgers Systems
}

\author{
Yan-ze Peng \\ School of Mathematics and Statistics, Wuhan University, Wuhan, 430072, China \\ Correspondence should be addressed to Yan-ze Peng, yanzepeng@163.com
}

Received 3 July 2009; Revised 10 December 2009; Accepted 23 December 2009

Recommended by Prabir Daripa

A $(2+1)$ dimensional Burgers equation and a coupled higher dimensional Burgers system is studied by the singular manifold method. The Bäcklund transformations are obtained. Some interesting exact solutions are given. Then localized structures, such as dromion and solitoff, are found, and their interaction properties are numerically studied. The fusion phenomena of two dromions, a dromion and a solitoff, are for the first time reported.

Copyright (c) 2009 Yan-ze Peng. This is an open access article distributed under the Creative Commons Attribution License, which permits unrestricted use, distribution, and reproduction in any medium, provided the original work is properly cited.

\section{Introduction}

The solitary wave solutions of $(1+1)$ dimensional PDEs have been studied quite well and widely applied in many fields of physics $[1,2]$. In $(2+1)$ dimensional, some significant nonlinear physical models such as the Kadomtsev-Petviashvili (KP) equation [3], DaveyStewartson (DS) equation [4] and so forth. have been established. And some special types of localized solutions, dromions and solitoffs, for example, are obtained for these higherdimensional models by means of different appropriates $[5,6]$. Dromions are exact, localized solutions of $(2+1)$ dimensional evolution equations and decay exponentially in all directions. Solitoffs constitute an intermediate state between dromions and plane solitons, since they decay exponentially in all directions except a preferred one. Although some generalized dromion and solitoff structures have been exposed [7, 8], the construction of localized excitations in $(2+1)$ dimensions is still a challenging and rewarding problem.

In this paper, we consider the construction of localized structures in a $(2+1)$ dimensional Burgers equation [9]:

$$
u_{t}+u_{x y}+u u_{y}+u_{x} \partial_{x}^{-1} u_{y}=0
$$


and a coupled higher-dimensional Burgers system of the form [10, 11]

$$
\begin{aligned}
& u_{t}=u_{x x}+u_{y y}+2 u u_{x}+2 v u_{y} \\
& v_{t}=v_{x x}+v_{y y}+2 u v_{x}+2 v v_{y} .
\end{aligned}
$$

Equation (1.1) reduces to the well-known Burgers equation when $y=x$. Recently, Kaya and Yokus [12] obtained some plane solitary wave solutions by a modified Adomian's decomposition method. However, localized structures of (1.1) and (1.2) have not yet been reported, to our knowledge.

The organization of the paper is as follows. In Section 2, a general functional separation solution containing two arbitrary functions is obtained for (1.1). Equation (1.2) is transformed into a single heat equation by a function transformation in Section 3. Exact solutions and localized structures are discussed in Section 4, and their interaction properties are numerically studied. The conclusion and discussion are given in Section 5.

\section{A General Solution to (1.1)}

Under the transformation $u_{y}=v_{x},(1.1)$ is converted into a set of couple of nonlinear partial differential equations:

$$
\begin{gathered}
u_{t}+u_{x y}+u u_{y}+u_{x} v=0 \\
u_{y}=v_{x} .
\end{gathered}
$$

According to the singular manifold method [13, 14], we truncate the Painlevé expansion of (2.1) at the constant level term

$$
\begin{aligned}
& u=\varphi^{-1} u_{0}+u_{1} \\
& v=\varphi^{-1} v_{0}+v_{1}
\end{aligned}
$$

where $\varphi$ is the singular manifold, and $\left\{u_{1}, v_{1}\right\}$ is an arbitrary seed solution of (2.1). Substituting (2.2) into (2.1) and equating the coefficients of like powers of $\varphi$ yield

$$
u_{0}=\varphi_{x}, \quad v_{0}=\varphi_{y}
$$

where $\varphi$ satisfies the equation

$$
\varphi_{t}+\varphi_{x y}+u_{1} \varphi_{y}+v_{1} \varphi_{x}=0
$$

which is called the singular manifold equation. Equations (2.2), (2.3), and (2.4) constitute an auto-Bäcklund transformation for (2.1) in terms of the singular manifold $\varphi$. If we take $u_{1}=\varphi$, $v_{1}=\partial_{x}^{-1} \varphi_{y}$, then

$$
u=\frac{\varphi_{x}}{\varphi}+\varphi
$$


where $\varphi$ satisfies

$$
\varphi_{t}+\varphi_{x y}+\varphi \varphi_{y}+\varphi_{x} \partial_{x}^{-1} \varphi_{y}=0
$$

Equations (2.5) and (2.6) are another auto-Bäcklund transformation for (1.1). If we take $u_{1}=$ $0, v_{1}=0$, the Cole-Hopf type transformation or hetero-Bäcklund transformation

$$
u=\frac{\varphi_{x}}{\varphi}
$$

where $\varphi$ satisfies

$$
\varphi_{t}+\varphi_{x y}=0
$$

is obtained for $(2+1)$-dimensional Burgers equation (1.1). Now, we take the special seed solution as

$$
u_{1}=0, \quad v_{1}=v_{1}(y, t)
$$

where $v_{1}(y, t)$ is an arbitrary function of indicated variables. It can be directly checked that (2.4) with (2.9) has the nonlinear separation solution

$$
\varphi=\mathrm{e}^{x} g(y, t)+h(y)
$$

with $g(y, t)$ and $h(y)$ being arbitrary functions of indicated variables if we take

$$
v_{1}=-\frac{g_{y}+g_{t}}{g}
$$

Thus, the direct calculation from (2.2), (2.3), (2.9), and (2.10) yields a general functional separation solution of $(1.1)$

$$
u=\frac{\mathrm{e}^{x} g}{\mathrm{e}^{x} g+h}
$$

with $g(y, t)$ and $h(y)$ being arbitrary functions of indicated variables. The solution generated this way involves two arbitrary functions of space and time variables without any restriction. This implies that we can study a large diversity of solution structures for the $(2+1)$ dimensional Burgers equation (1.1) by selecting appropriately these arbitrary functions in (2.12). It is necessary to point out that the $(2+1)$-dimensional Burgers equation (1.1) possesses some special types of localized coherent structures for the following potential field:

$$
w \equiv u_{y}=\frac{g_{y} h-g h_{y}}{\left(\mathrm{e}^{x / 2} g+\mathrm{e}^{-x / 2} h\right)^{2}}
$$

rather than the physical field $u$ itself. 


\section{The Linearization of $(1.2)$}

Through a similar analysis, we obtain the following auto-Bäcklund transformation of (1.2):

$$
\begin{aligned}
& u=\frac{\varphi_{x}}{\varphi}+u_{1}, \\
& v=\frac{\varphi_{y}}{\varphi}+v_{1},
\end{aligned}
$$

where $\left\{u_{1}, v_{1}\right\}$ is the seed solution to (1.2) and $\varphi$ satisfies the equation

$$
\varphi_{t}=\varphi_{x x}+\varphi_{y y}+2 u_{1} \varphi_{x}+2 v_{1} \varphi_{y}
$$

with the constraint $u_{1 y}=v_{1 x}$. Taking the seed solution $u_{1}=\varphi, v_{1}=\partial_{x}^{-1} \varphi_{y}$, one obtains a new Bäcklund transformation (2.5), along with $v=\varphi_{y} / \varphi+\partial_{x}^{-1} \varphi_{y}$, for (1.2) with $\varphi$ satisfying

$$
\varphi_{t}=\varphi_{x x}+\varphi_{y y}+2 \varphi \varphi_{x}+2 \varphi_{y} \partial_{x}^{-1} \varphi_{y}
$$

If taking the trivial seed solution $u_{1}=v_{1}=0$, the Cole-Hopf type transformation

$$
\begin{aligned}
& u=\frac{\varphi_{x}}{\varphi}, \\
& v=\frac{\varphi_{y}}{\varphi},
\end{aligned}
$$

with $\varphi$ satisfying

$$
\varphi_{t}=\varphi_{x x}+\varphi_{y y}
$$

is obtained for (1.2). Thus, the nonlinear equation (1.2) is linearized into (3.5) by the transformation (3.4). Through (3.5), one may obtain many interesting solution structures of (1.2). However, the coupled higher-dimensional Burgers system (1.2) possesses special types of localized coherent structures for the potential field $w \equiv u_{y}$, rather than the physical field $u$ or $v$ itself.

\section{Special Exact Solutions and Localized Structures for (1.1) and (1.2)}

By selecting appropriately these arbitrary functions in (2.12), we can study many interesting solution structures for the $(2+1)$-dimensional Burgers equation (1.1). Two new cases are considered as an illustrative example, and others can be obtained in a similar way to that in $[9,13]$.

Case 1. We have $g=\exp \left[\tanh \left(l_{1} y-\omega_{1} t\right)\right]+\exp \left[\tanh \left(l_{2} y-\omega_{2} t\right)\right] \equiv \exp \left(\tanh \xi_{1}\right)+\exp \left(\tanh \xi_{2}\right)$, $h=\exp [\tanh (l y)]+A \equiv \exp (\tanh \xi)+A$. 


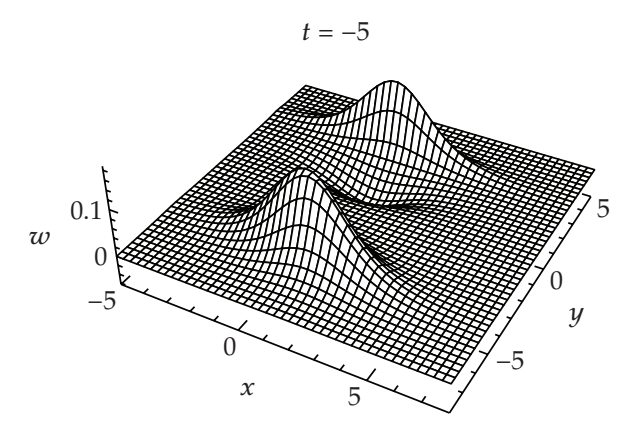

(a)

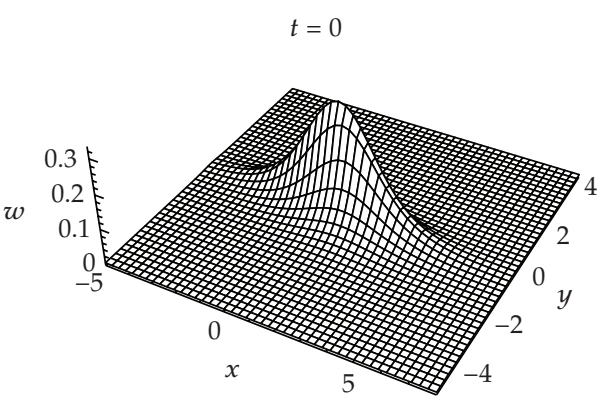

(b)

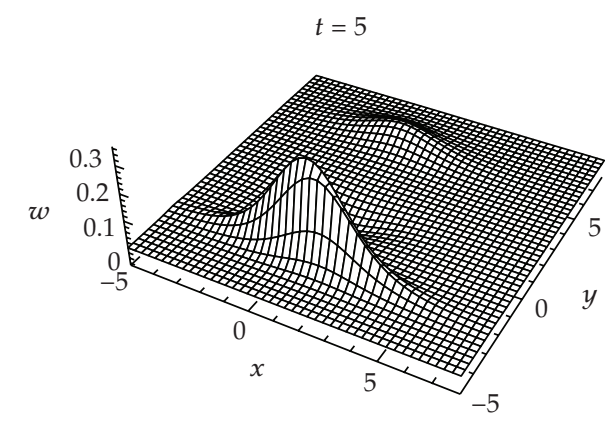

(c)

Figure 1: The evolution of three dromions-like for (4.2).

From (2.12), one gets an exact solution of (1.1)

$$
u=\left\{\ln \left[\exp (x)\left(\exp \left(\tanh \xi_{1}\right)+\exp \left(\tanh \xi_{2}\right)\right)+\exp (\tanh \xi)+A\right]\right\}_{x} .
$$

It follows from (2.13) that

$$
w=\left\{\ln \left[\exp (x)\left(\exp \left(\tanh \xi_{1}\right)+\exp \left(\tanh \xi_{2}\right)\right)+\exp (\tanh \xi)+A\right]\right\}_{x y^{\prime}}
$$

which is a three-dromion-like structure (two dromions-like and one anti-dromion-like), ant its evolution with time is shown in Figure 1 with parameters $l_{1}=1, \omega_{1}=1, l_{2}=2, \omega_{2}=$ $-1, l=1, A=4$, and $t=-5,0,5$, respectively. One can easily see that the interaction of three dromions-like is inelastic.

Case 2. We have $g=\exp \left(\operatorname{sech} \xi_{1}\right)+\exp \left(\operatorname{sech} \xi_{2}\right), h=\exp (\operatorname{sech} \xi)+A$.

Another exact solution of (1.1) reads

$$
u=\left\{\ln \left[\exp (x)\left(\exp \left(\operatorname{sech} \xi_{1}\right)+\exp \left(\operatorname{sech} \xi_{2}\right)\right)+\exp (\operatorname{sech} \xi)+A\right]\right\}_{x}
$$

The corresponding localized structure is

$$
w=\left\{\ln \left[\exp (x)\left(\exp \left(\operatorname{sech} \xi_{1}\right)+\exp \left(\operatorname{sech} \xi_{2}\right)\right)+\exp (\operatorname{sech} \xi)+A\right]\right\}_{x y^{\prime}}
$$


which is a four dromions-like solution. Its evolution figures are very similar to those in Case 1 and thus omitted. In what follows, the stress is played on solution structures for the coupled higher-dimensional Burgers system (1.2). For the exact solution (3.4) of (1.2), the function $\varphi$ must satisfies (3.5). Some meaningful cases are considered.

Case 3. We have $\varphi=1+\mathrm{e}^{k x+l y+\left(k^{2}+l^{2}\right) t}$.

From (3.4), one obtain

$$
\begin{aligned}
& u=\frac{1}{2} k\left[1+\tanh \frac{1}{2}\left(k x+l y+\left(k^{2}+l^{2}\right) t\right)\right], \\
& v=\frac{1}{2} l\left[1+\tanh \frac{1}{2}\left(k x+l y+\left(k^{2}+l^{2}\right) t\right)\right],
\end{aligned}
$$

a shock wave solution of (1.2).

Case 4. We have $\varphi=1+\mathrm{e}^{k x+k^{2} t}+\mathrm{e}^{l y+l^{2} t}$.

It follows from (3.4) that

$$
\begin{aligned}
& u=\frac{k \mathrm{e}^{k x+k^{2} t}}{1+\mathrm{e}^{k x+k^{2} t}+\mathrm{e}^{l y+l^{2} t}}, \\
& v=\frac{l \mathrm{e}^{l y+l^{2} t}}{1+\mathrm{e}^{k x+k^{2} t}+\mathrm{e}^{l y+l^{2} t}},
\end{aligned}
$$

a new exact solution for (1.2).

Case 5. We have $\varphi=1+\mathrm{e}^{k x+k^{2} t}+\mathrm{e}^{l y+l^{2} t}+A \mathrm{e}^{k x+l y+\left(k^{2}+l^{2}\right) t}$.

From (3.4), one gets another new exact solution for (1.2)

$$
\begin{aligned}
& u=\frac{k \mathrm{e}^{k x+k^{2} t}+k A \mathrm{e}^{k x+l y+\left(k^{2}+l^{2}\right) t}}{1+\mathrm{e}^{k x+k^{2} t}+\mathrm{e}^{l y+l^{2} t}+A \mathrm{e}^{k x+l y+\left(k^{2}+l^{2}\right) t}}, \\
& v=\frac{l \mathrm{e}^{l y+l^{2} t}+l A \mathrm{e}^{k x+l y+\left(k^{2}+l^{2}\right) t}}{1+\mathrm{e}^{k x+k^{2} t}+\mathrm{e}^{l y+l^{2} t}+A \mathrm{e}^{k x+l y+\left(k^{2}+l^{2}\right) t}} .
\end{aligned}
$$

Note that $k, l, A$ are arbitrary constants, where $A$ guarantees that the expression (4.7) has no singularity. For exact solution (4.7), the corresponding localized structure is

$$
w=\left\{\ln \left[1+\mathrm{e}^{k x+k^{2} t}+\mathrm{e}^{l y+l^{2} t}+A \mathrm{e}^{k x+l y+\left(k^{2}+l^{2}\right) t}\right]\right\}_{x y} .
$$

When $A \neq 0,(4.8)$ is a one dromion structure. Its typical spatial structure is depicted in Figure 2 with the parameters $k=1, l=1, t=0$ and $A=2$. When $A=0,(4.8)$ is a one solitoff structure. Its typical spatial structure is shown in Figure 3 with the parameters $k=1, l=-1, t=0$ and $A=0$. Now, we study two kinds of interesting nonlinear interaction 


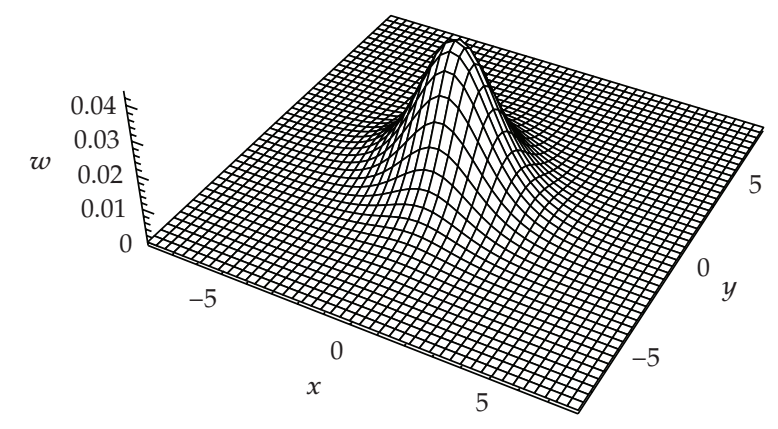

Figure 2: A typical spatial structure for (4.8) with $A=2$.

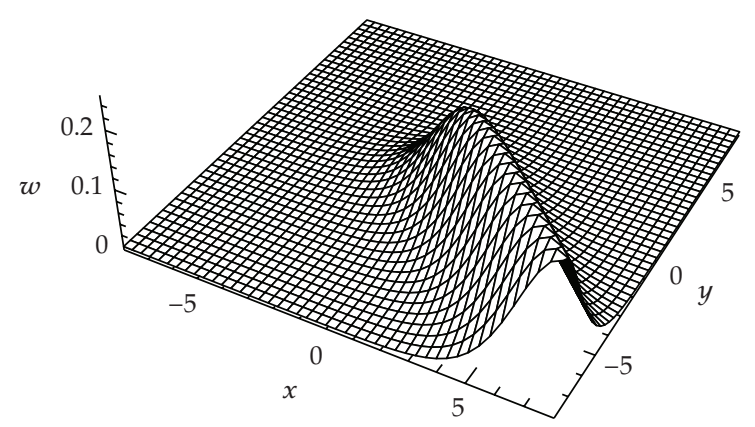

Figure 3: A typical spatial structure for (4.8) with $A=0$.

phenomena for localized structures of (1.2), which are not reported yet in literature to our knowledge.

Case 6. We have $\varphi=1+\mathrm{e}^{k x+k^{2} t}+\mathrm{e}^{l_{1} y+l_{1}^{2} t}+\mathrm{e}^{l_{2} y+l_{2}^{2} t}+A_{1} \mathrm{e}^{k x+l_{1} y+\left(k^{2}+l_{1}^{2}\right) t}+A_{2} \mathrm{e}^{k x+l_{2} y+\left(k^{2}+l_{2}^{2}\right) t}+$ $A_{3} \mathrm{e}^{\left(l_{1}+l_{2}\right) y+\left(l_{1}+l_{2}\right)^{2} t}$.

In this case, the localized structure of (1.2) reads

$$
\begin{gathered}
w=\left\{\operatorname { l n } \left[1+\mathrm{e}^{k x+k^{2} t}+\mathrm{e}^{l_{1} y+l_{1}^{2} t}+\mathrm{e}^{l_{2} y+l_{2}^{2} t}+A_{1} \mathrm{e}^{k x+l_{1} y+\left(k^{2}+l_{1}^{2}\right) t}\right.\right. \\
\left.\left.+A_{2} \mathrm{e}^{k x+l_{2} y+\left(k^{2}+l_{2}^{2}\right) t}+A_{3} \mathrm{e}^{\left(l_{1}+l_{2}\right) y+\left(l_{1}+l_{2}\right)^{2} t}\right]\right\}_{x y^{\prime}}
\end{gathered}
$$

which is a two-dromion-like (a dromion-like and an anti-dromion-like) structure. And its evolution is illustrated in Figure 4 with the parameters $k=1, l_{1}=-1, l_{2}=2, A_{1}=1, A_{2}=$ $2, A_{3}=3$ and $t=-5,0,5$, respectively. From the figures, we see that two dromions-like are fused into a dromion-like after their interaction.

Case 7. We have $\varphi=1+\mathrm{e}^{k x+k^{2} t}+\mathrm{e}^{l_{1} y+l_{1}^{2} t}+A_{1} \mathrm{e}^{k x+l_{1} y+\left(k^{2}+l_{1}^{2}\right) t}+A_{2} \mathrm{e}^{k x+l_{2} y+\left(k^{2}+l_{2}^{2}\right) t}+A_{3} \mathrm{e}^{\left(l_{1}+l_{2}\right) y+\left(l_{1}+l_{2}\right)^{2} t}$. 


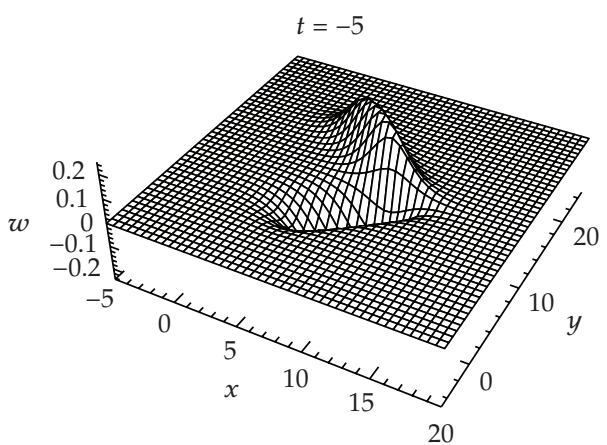

(a)

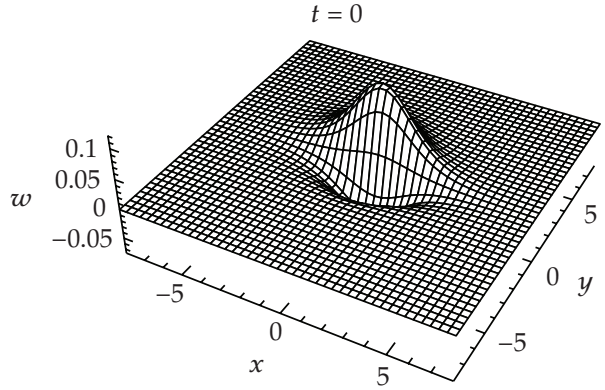

(b)

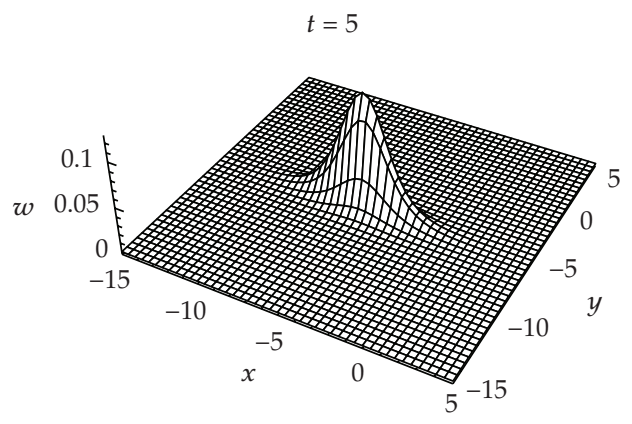

(c)

Figure 4: The fussion phenomenon of two dromions-like for (4.9).

This case is obtained from the above one through dropping the third exponential term. Thus, the localized structure is

$$
\begin{aligned}
w=\{\ln [ & 1+\mathrm{e}^{k x+k^{2} t}+\mathrm{e}^{l_{1} y+l_{1}^{2} t}+A_{1} \mathrm{e}^{k x+l_{1} y+\left(k^{2}+l_{1}^{2}\right) t} \\
& \left.\left.+A_{2} \mathrm{e}^{k x+l_{2} y+\left(k^{2}+l_{2}^{2}\right) t}+A_{3} \mathrm{e}^{\left(l_{1}+l_{2}\right) y+\left(l_{1}+l_{2}\right)^{2} t}\right]\right\}_{x y^{\prime}}
\end{aligned}
$$

which is a combination structure of one dromion-like and one solitoff-like. And its evolution is shown in Figure 5 with the same parameter values as those in Figure 4. One can easily see from the figures that a dromions-like and a solitoff-like are fused into a solitoff-like after their interaction.

\section{Conclusion and Discussion}

We have obtained auto-Bäcklund transformations, Cole-Hopf ones, and a general functional separation solution containing two arbitrary functions for the $(2+1)$-dimensional Burgers equation by means of choosing different seed solutions in the singular manifold method, and a coupled higher-dimensional Burgers system (1.2) has been linearized. It is pointed out that the equations of interest possess some special types of localized coherent structures for the potential field $w \equiv u_{y}$ rather than the physical field $u$ or $v$ itself. For (1.1), we find that the 


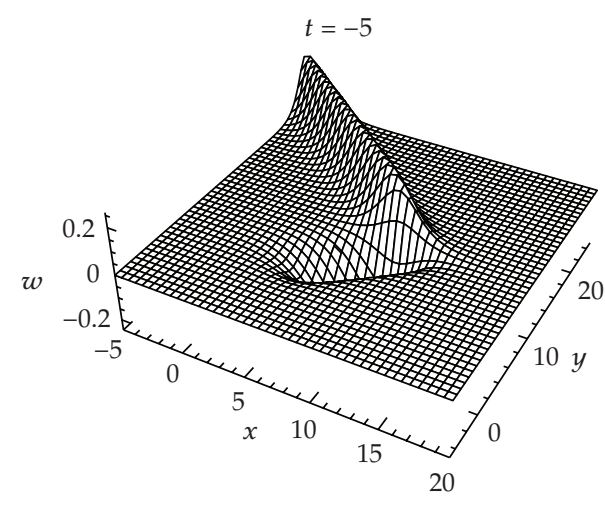

(a)

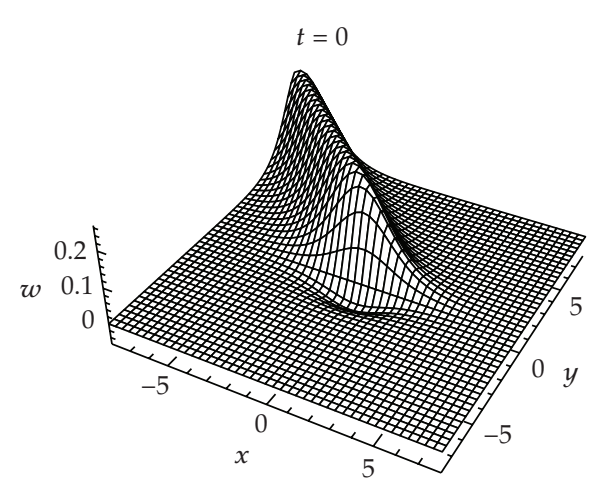

(b)

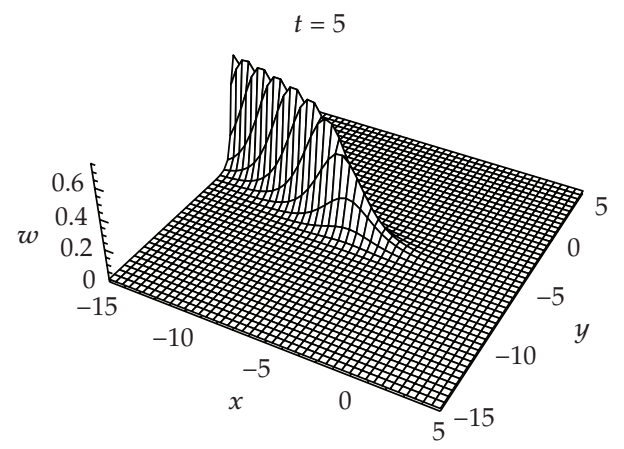

(c)

Figure 5: The fussion phenomenon of a dromion-like and a solitoff-like for (4.10).

interaction of three dromions-like is inelastic. As far as (1.2) is concerned, it is found that two dromions-like can be fused into one while a dromion-like and a solitoff-like can be fused into a solitoff-like, which are not reported previously in the literature.

The singular manifold method is a powerful tool for obtaining exact solutions of nonlinear PDEs. Usually, the seed solution is taken as the trivial one in order to get the solution of the singular manifold equation. Due to the introduction of two arbitrary functions in our singular manifold function, one can study a variety of solution structures by choosing appropriately these arbitrary functions. Even more, the equation of interest may be linearized.

\section{Acknowledgment}

This work is supported by Research Fund for the Doctoral Program of Higher Education of China (no. 20070486094).

\section{References}

[1] I. Loutsenko and D. Roubtsov, "Critical velocities in exciton superfluidity," Physical Review Letters, vol. 78, no. 15, pp. 3011-3014, 1997.

[2] M. Tajiri and H. Maesono, "Resonant interactions of drift vortex solitons in a convective motion of a plasma," Physical Review E, vol. 55, no. 3, pp. 3351-3357, 1997. 
[3] B. B. Kadomtsev and V. I. Petviashvili, "On the stability of solitary waves in weakly dispersive media," Soviet Physics Doklady, vol. 15, pp. 539-541, 1970.

[4] A. Davey and K. Stewartson, "On three-dimensional packets of surface waves," Proceedings of the Royal Society A, vol. 338, pp. 101-110, 1974.

[5] A. S. Fokas and P. M. Santini, "Dromions and a boundary value problem for the Davey-Stewartson 1 equation," Physica D, vol. 44, no. 1-2, pp. 99-130, 1990.

[6] J. Hietarinta, "One-dromion solutions for generic classes of equations," Physics Letters A, vol. 149, no. 2-3, pp. 113-118, 1990.

[7] Y. Z. Peng, "Periodic waves and periodic solitons and their interactions for a (2+1)-dimensional KdV equation," Progress of Theoretical Physics, vol. 113, no. 5, pp. 927-933, 2005.

[8] Y. Z. Peng and E. V. Krishnan, "The singular manifold method and exact periodic wave solutions to a restricted BLP dispersive long wave system," Reports on Mathematical Physics, vol. 56, no. 3, pp. 367-378, 2005.

[9] J. M. Burgers, The Nonlinear Diffusion Equation, Reidel, Dordrecht, The Netherlands, 1974.

[10] K. M. Tamizhmani and P. Punithavathi, "Similarity reductions and Painlevé property of the coupled higher-dimensional Burgers' equation," International Journal of Non-Linear Mechanics, vol. 26, no. 3-4, pp. 427-438, 1991.

[11] M. Salerno, "On the phase manifold geometry of the two-dimensional Burgers equation," Physics Letters A, vol. 121, no. 1, pp. 15-18, 1987.

[12] D. Kaya and A. Yokus, "A decomposition method for finding solitary and periodic solutions for a coupled higher-dimensional Burgers equations," Applied Mathematics and Computation, vol. 164, no. 3, pp. 857-864, 2005.

[13] J. Weiss, M. Tabor, and G. Carnevale, "The Painlevé property for partial differential equations," Journal of Mathematical Physics, vol. 24, no. 3, pp. 522-526, 1983.

[14] P. R. Gordoa, A. Pickering, and J. Prada, "Non-isospectral scattering problems and truncation for hierarchies: Burgers and dispersive water waves," Physica A, vol. 345, no. 1-2, pp. 35-47, 2005. 


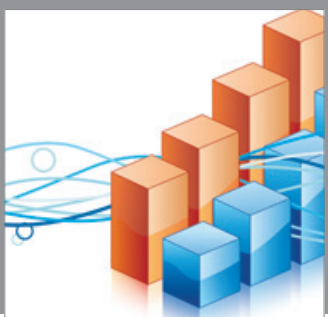

Advances in

Operations Research

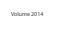

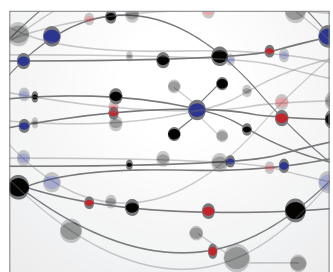

\section{The Scientific} World Journal
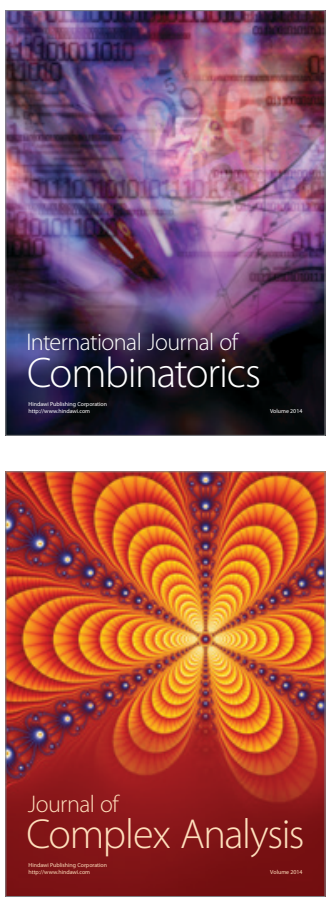

International Journal of

Mathematics and

Mathematical

Sciences
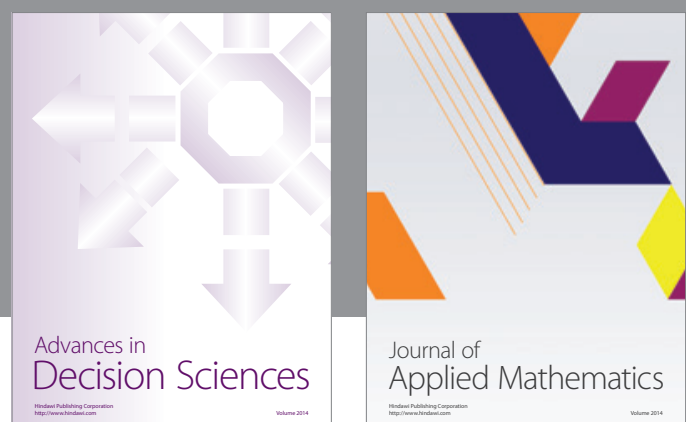

Journal of

Applied Mathematics
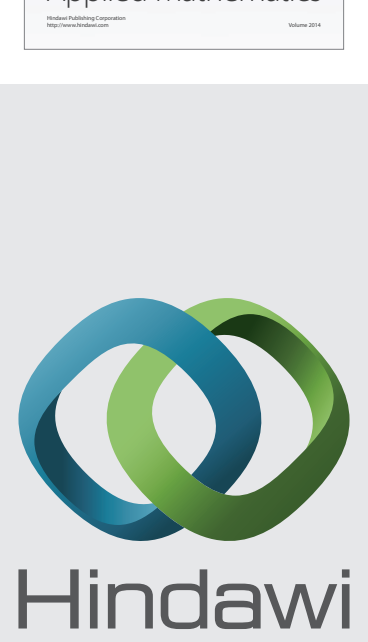

Submit your manuscripts at http://www.hindawi.com
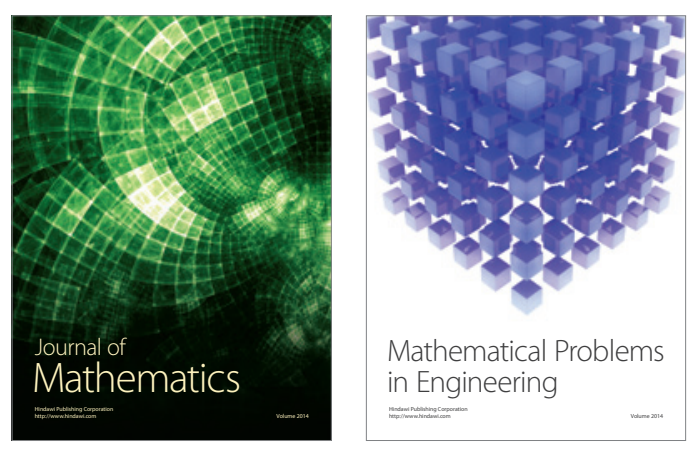

Mathematical Problems in Engineering
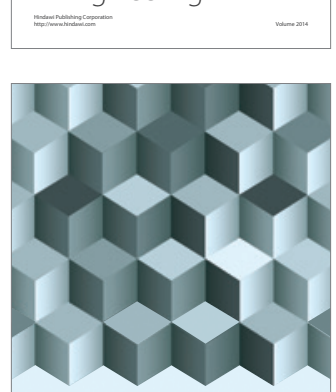

Journal of

Function Spaces
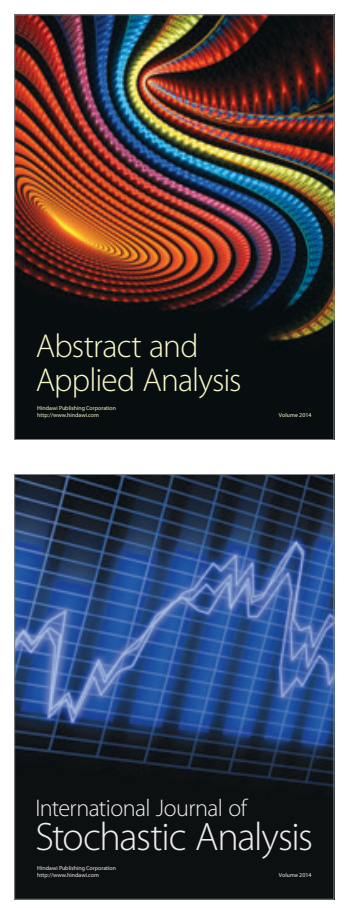

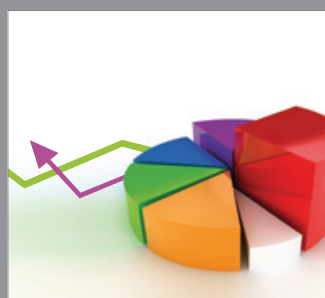

ournal of

Probability and Statistics

Promensencen
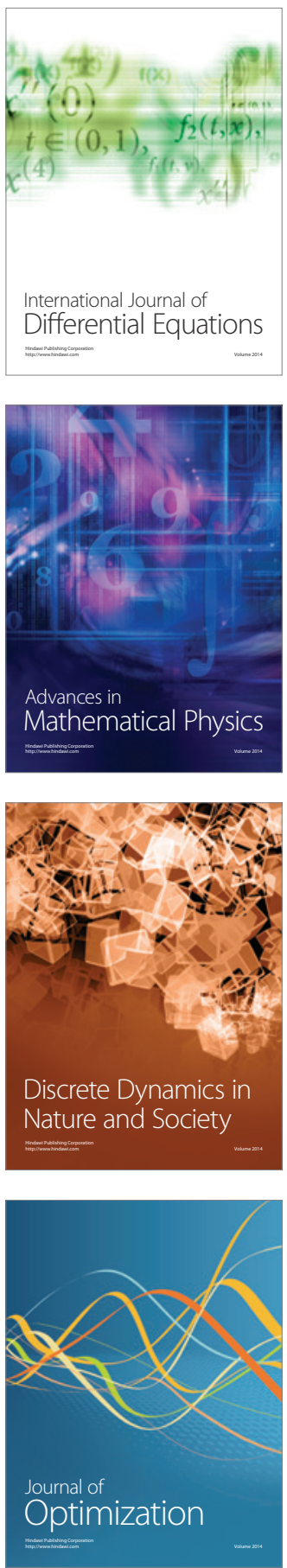